\title{
ITERATIVE ALGORITHM FOR MICROWAVE IMAGING USING THE CGM-FFT AND THE PSEUDOINVERSE METHOD
}

Jordi J. Mallorqui", Vicenç Llorens

Grup A.M.R., Dept. of Signal Theory and Communications, ETSETB.

P.O. Box 30002, 08080 Barcelona, SPAIN

\section{ABSTRACT}

A spatial iterative method for the reconstruction of the complex permittivity of inhomogeneous lossy dielectric objects with arbitrary shape from their scattered electric field is shown. Starting from the integral representation of the electric field and by means of the method of the moments (MoM) and a first order linearization of the resulting expressions, the integral equations are converted into matrix equations building an iterative scheme. This technique has been developed for the bidimensional TM case with cylindrical geometry. The advantages and limitations of this algorithm are also discussed.

\section{INTRODUCTION}

The microwave tomographic systems consists of a circular array of antennas placed around the object. The antennas act as sources and receivers to produce a complete scan of the object. With one of the antennas radiating and the remaining elements of the array receiving sequentially a view is obtained. The measurement process ends when all the views are adquired. Figure 1 shows the measurement scheme and the notation used on the paper.

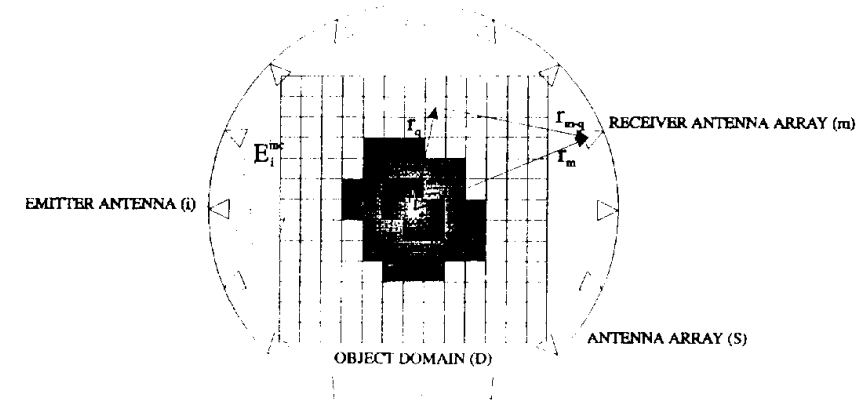

Fig. 1. Gesmetry of the problem: antennat arriay and object domain.

The Helmoltz equation relates the harmonic incident field, the contrast function and the Green's function with the scatlered and diffracted fields. For the diffracted 
increment is found by using the pseudoinverse method, implying the quadratic minimization of the error.

All the steps of the algorithm can be summarized as follows:

0 . Initial guess, the shape of the object can be obtained by using a first order algorithm under the Born approximation and its average permittivity can be supplied to the algorithm or calculated $[1]$.

1. The total field is obtained from the present contrast function by using the CGMFFT algorithm or the LU decomposition [2].

2. From the diffracted field over the object domain the scattered field on the array is calculated.

3. The error between the calculated and measured scattered fields is obtained.

4. From (10) the increment of contrast functions is calculated.

5. The contrast function is updated.

6. If the quadratic error between calculated and measured scattered fields descends up to a mark, the process is stopped. Otherwise the iterative algorithm goes to step 1 .

For highly inhomogeneous bodies the inversion of (10) can present stability problems. In order to improve the stability of the inversion, the Tikhonov regularization has been implemented [3].

\section{RESULTS}

A reconstruction of a realistic model of a human neck is presented. On the left side of the figure the contrast model of the neck is shown and on the right side the final reconstructed image after 17 iterations. The working frequency is $1 \mathrm{GHz}$, the embedding medium is water and the neck is 3.7 wavelengths wide. The inner permittivity distribution can perfectly seen and the reconstructed image gives the real values of contrast. The algorithm has also been tested with a thorax mode and experimental results from a microwave tomographic prototype [1] with excellent results.

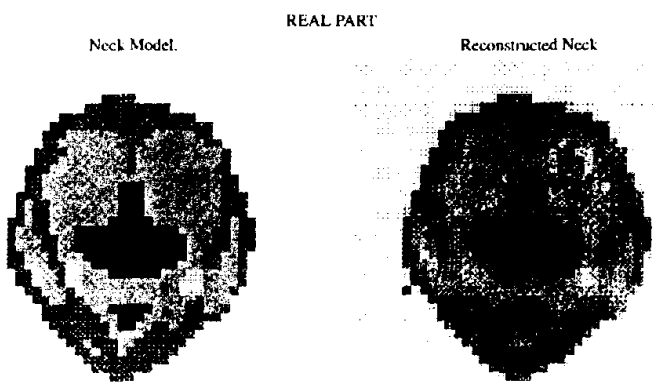




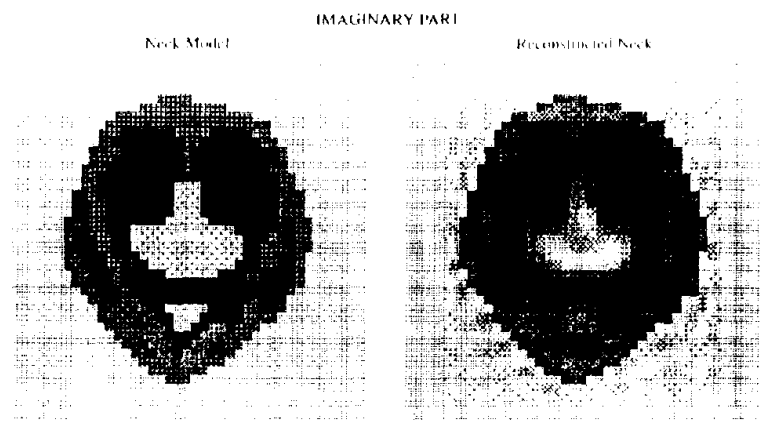

\section{CONCLUSIONS}

An iterative algorithm for microwave imaging has been presented. The final images show the correct contrast values, that means a significant improvement with respect to the Born approximation to the inverse electromagnetic problem.

\section{ACKNOWLEDGEMENT}

This work has been sponsored by the Comisión Interministerial de Ciencia y Tecnología (CICYT) under Grant TIC93-0518.

\section{REFERENCES}

[1] A. Broquetas, J.Romeu, J.M. Rius, A.R. Elías Fusté, A.Cardama, L. Jofre, "Cylindrical Geometry: A Further Step in Active Micriwave Tomography", IEEE Trans. on MTT, Vol. 39, $\mathrm{N}^{\mathrm{o}}$ 5, pp. 836-844, May 1991.

[2] M. Vaquer, J.J.Mallorquí, A. Cardama, L1. Jofre, "Bidimensional Scattering of TM and TE Polarized Waves by Dielectric Bodies Using The C.G.-F.F.T. Method; Application to Mobile Communication Systems". Proceedings of the $23^{\text {m }}$ European Microwave Conference, Madrid 6-9 Sept. 1993, pp. 409-411.

[3] Tapan K. Sarkar, D.D. Wiener, V.K. Jain, "Some Considerations Dealing With Inverse Problem". IEEE Trans. on AP, Vol 29, N"2. pp. 373-379, March 1981.

14] Nadine Joachinowice, C. Pichot, J.P. Hugonin "Inverse Scattering: An iterative Numerical Methol for Electromagnetic Imaging" III:E Trans. on AP, Vol. $N^{\prime \prime} 12$. p. 1742 1752, Dec. 1991. 